\title{
Discovery of parabolic SNAP microresonators produced in fibre tapering
}

Gabriella Gardosi, Sajid Zaki, Dashiell L. P. Vitullo, Kirill Tokmakov, Michael Brodsky, et al.

Gabriella Gardosi, Sajid Zaki, Dashiell L. P. Vitullo, Kirill Tokmakov, Michael Brodsky, Misha Sumetsky, "Discovery of parabolic SNAP microresonators produced in fibre tapering ," Proc. SPIE 10681, Micro-Structured and Specialty Optical Fibres V, 106810B (9 May 2018); doi: 10.1117/12.2307373 


\title{
Discovery of Parabolic SNAP Microresonators Produced in Fibre Tapering
}

\author{
Gabriella Gardosi*a, Sajid Zaki ${ }^{\mathrm{a}}$, Dashiell L. P. Vitullo ${ }^{\mathrm{a}}$, Kirill Tokmakov ${ }^{\mathrm{a}}$, Michael Brodsky ${ }^{\mathrm{b}}$ \\ and Misha Sumetsky ${ }^{\mathrm{a}}$ \\ ${ }^{a}$ Aston Institute of Photonic Technologies, Aston Expressway, Birmingham, B4 7ET UK \\ ${ }^{\mathrm{b}}$ U.S. Army Research Laboratory, Adelphi, MD, 20783-1197 USA
}

\begin{abstract}
We present a novel method based on optical fibre tapering for fabrication of Surface Nanoscale Axial Photonics (SNAP) devices with parabolic profiles with an unprecedentedly large number of axial eigenmodes. Tapering of a commercial $125 \mu \mathrm{m}$ single-mode optical fibre to a $30 \mu \mathrm{m}$ diameter waist by laser brushing creates a SNAP bottle microresonator with parabolic radius variation in the centre of the tapered region. Ideal parabolic resonators should demonstrate equal spacing between resonances. Our spectral measurement of the parabolic profile shows spacing of $\sim 6 \mathrm{GHz}$ with $10 \%$ deviation over a bandwidth of $2.5 \mathrm{THz}$ containing up to 400 axial eigenfrequencies. This new discovery for the creation of SNAP parabolic microresonator devices is important for fabrication of miniature delay lines, buffers and frequency comb generators. Characterisation of our exemplar microresonators is briefly explored, particularly for broadband frequency comb generators which require equidistant frequency spacing. Further investigations include scaling of the parabolic feature with tapering process parameters, repeatability testing, and the fabrication of more complex shapes.
\end{abstract}

Keywords: Whispering gallery mode, parabolic profile, frequency comb generation, Surface Nanoscale Axial Photonics, harmonic potential, bottle resonator, taper

\section{INTRODUCTION}

Optical microresonators with equidistant spacing of eigenfrequencies are important for several applications including fabrication of miniature frequency comb generators ${ }^{1-4}$, dispersion-free optical delay lines and buffers ${ }^{5,6}$. The performance of these devices is ensured by their ultralow losses (high Q-factor) and high fabrication precision. Optical bottle resonators with parabolic effective radius variation (ERV) ${ }^{7}$ are arguably the simplest kind of microresonators with equidistant eigenfrequencies. Their fabrication based on Surface Nanoscale Axial Photonics (SNAP) technology has achieved unparalleled subangstrom precision in $\mathrm{ERV}^{8,9}$. SNAP microresonators with semiparabolic profiles fabricated previously involved complex procedure of precisely controlled $\mathrm{CO}_{2}$ laser exposure along a fibre. Furthermore, their characteristic ERV was of the order of 10 nanometres, which is not sufficient for fabrication of frequency comb generators which are broadband and low repetition rate simultaneously ${ }^{10}$.

Previously Birks, Knight and Dimmick investigated the uniformity of biconical taper waists fabricated by flame brushing in order to test their theory of taper shape ${ }^{11}$. Their theory predicted that the taper radius should be uniform near the taper waist, and measurement with whispering gallery mode (WGM) spectroscopy method developed in ${ }^{12}$, found less than $2 \%$ variation of the taper waist radius. This small variation is negligible for most major applications. However, nanometre scale radius deformations observed in ${ }^{7}$ are sufficient to localize light and form SNAP microresonators. Here we report the discovery of bottle microresonators formed through the tapering process with a parabolic longitudinal profile and $\sim 100 \mathrm{~nm}$ ERV height containing hundreds of uniformly-spaced axial modes.

*gardosig@aston.ac.uk

Micro-Structured and Specialty Optical Fibres V, edited by Kyriacos Kalli, Alexis Mendez,

Christian-Alexander Bunge, Proc. of SPIE Vol. 10681, 106810B - (C) 2018 SPIE

CCC code: $0277-786 \mathrm{X} / 18 / \$ 18 \cdot$ doi: $10.1117 / 12.2307373$

Proc. of SPIE Vol. 10681 106810B-1 


\section{OPTICAL MICRORESONATORS}

Conventional optical resonators were developed with highly reflective surfaces to confine light within a cavity. The goal to achieve miniaturisation of this design led to the development of multiple platforms all of which sought to overcome the challenge of fabrication precision and reduction of attenuation losses.

It is convenient to describe light in an optical resonator in terms of its discrete spatial eigenmodes. These orthogonal electromagnetic oscillations fulfill resonance conditions at corresponding resonant frequencies (eigenfrequencies), which depend upon the physical characteristics of the cavity. For the simplest case, a 1D cavity of length, L,

$$
q\left(\frac{\lambda_{r}}{2}\right)=L
$$

where $q$ is axial or longitudinal mode number (whose fundamental corresponds to $q=1$ ) and $\lambda_{r}$ is the resonant wavelength.

Modes of 3D cavities, such as bottle resonators, of course have more degrees of freedom. For example, a cylindrical resonator, considered in this paper (whose fundamental corresponds to $q=0$ ), is completely described with axial, azimuthal, radial, and polarization quantum numbers $q, m, p$, and $s$, respectively.

The spacing between adjacent eigenfrequencies is known as the free spectral range (FSR),

$$
\Delta \lambda_{a x}^{N}=\lambda_{q, m, p, s}-\lambda_{q+1, m, p, s}
$$

Of particular interest are devices with equidistant eigenfrequencies, for applications in delay lines, optical buffers and frequency comb generators. Potentials that yield equally spaced modes were discussed in ${ }^{6}$, the most popular profile being parabolic, owing to its simplicity. Microresonators with parabolic profiles have been developed in, photonic crystal cavities $^{13}$, semiconductor microtube microresonators ${ }^{14}$ and bottle microresonators ${ }^{7,8}$.

Ideal optical resonators experience no losses. This ideal is approached in an actual resonator when it has a very large Qfactor, which is a figure of merit defined by,

$$
Q=2 \pi \frac{\text { stored energy }}{\text { energy loss per round trip }} \text {. }
$$

This can be measured with an optical spectrum analyser using,

$$
Q=\frac{\lambda_{r}}{\text { FWHM }}
$$

where FWHM is full width half maximum of the resonant spectral line.

\section{SNAP MICRORESONATORS}

The principal SNAP method marries the near-grazing incident reflections of WGMs at the fibre surface with the exploitation of optical fibre's intrinsic tension caused by its drawing manufacture, to fabricate photonic devices at the surface of a fibre with unparalleled precision ${ }^{8,9}$. Construction of SNAP bottle microresonators usually consist of exposures to a $\mathrm{CO}_{2}$ laser beam, which locally anneals the optical fibre and releases this tension, see Fig. 1 (a). This results in fabrication of single and coupled bottle microresonators with an ERV height typically up to $10 \mathrm{~nm}$, low loss $\left(10^{-4} \mathrm{~dB} / \mathrm{cm}\right)$ and high $\mathrm{Q}\left(>10^{7}\right)^{15}$.

These devices are characterised by orienting a microfibre (the waist of an input-output probe taper) tangent to the target SNAP fibre, see Fig. 1 (b). The light sent through the microfibre via the evanescent field couples to WGMs that circulate near the surface of the target fibre. Power transmission through the probe taper dips at the resonant frequencies, sensitive to the ERV. Thus, by translating the microfibre along the length of the fibre under test the variation of radius is mapped.

WGMs are confined by a circular boundary, whose field is described in cylindrical coordinates as,

$$
E_{m, p, q}(\rho, \varphi, z)=\exp (i m \varphi) \cdot U_{m, p}\left(\frac{\rho}{r(z)}\right) \cdot \Psi_{m, p, q}(z) .
$$


For a WGM bottle resonator light has slow axial propagation between turning points $z_{1}$ and $z_{2}$, modelled by the 1D stationary Schrödinger equation ${ }^{16}$,

$$
\Psi_{z z}+[E(\lambda)-V(z)] \Psi=0 .
$$

The potential $V(z)$ is controlled by small variation of fibre radius with $n_{r}$ refractive index, $V(z)=-\kappa^{2} \frac{\Delta r(z)}{r_{w}}$ where $\kappa=2^{\frac{3}{2}} \pi n_{r} / \lambda_{r}$.

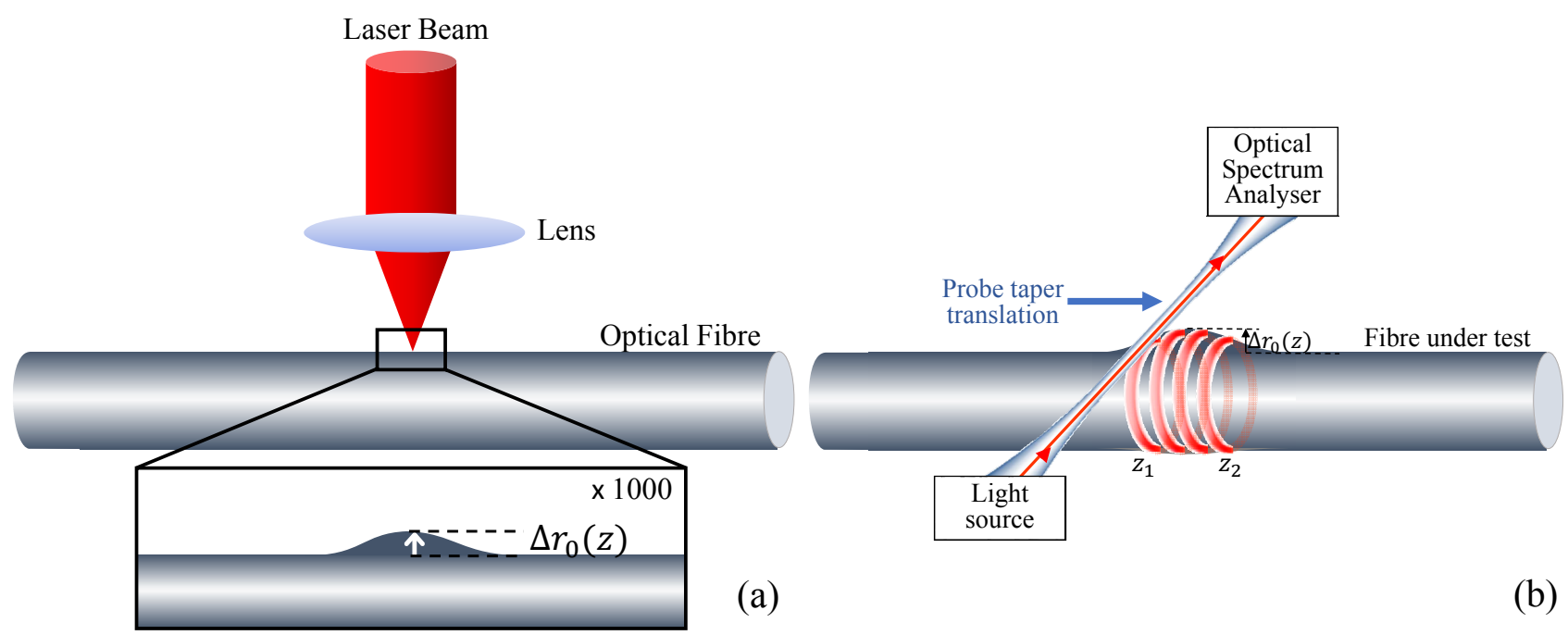

Figure 1. (a) Usual fabrication of SNAP bottle microresonator via $\mathrm{CO}_{2}$ laser beam exposure. Inset $\times 1000$ zoom of fabricated resonator on fibre surface with ERV, $\Delta r_{0}(z)$. (b) Characterisation of the microresonator through WGM spectroscopy delivered by the probe taper at positions along the fibre under test. Excited WGMs in the resonator are shown in red between turning points, $z_{1}$ and $z_{2}$, and are detected as transmission losses at the OSA.

\section{FABRICATION OF SNAP BOTTLE MICRORESONATOR WITH PARABOLIC PROFILE BY TAPERING}

Tapering is the process of heating and stretching an optical fibre to a predetermined diameter. A range of wellestablished fabrication methods exist for fibre tapering which all consist of a heating element, and fibre pulling with precise control. These tapered fibres are used widely in laboratory-based and commercial photonics applications for coupling and sensing applications.

\subsection{Taper properties}

As shown in Fig. 2, the thinnest region is referred to as the taper waist. If the length that is heated during the tapering process is held constant while the fibre is pulled, the model of Birks et al. predicts a taper waist of uniform diameter ${ }^{11}$. The taper transitions show the rate of change from the waist to the unstretched fibre, which should be adiabatically slow to maintain high transmission of light through the tapered region.

\subsection{Fabrication}

For tapering, our experimental setup employs a laser brushing technique using $\mathrm{CO}_{2}$-laser-heated (Synrad 48-2) sapphire microfurnace, as shown in Fig. 3. The length of the microfurnace hot zone can be varied by adjusting the laser power output and the beam's focal parameters ${ }^{17}$. This method benefits from setup simplicity and from having no naked flame so that the introduction of $\mathrm{OH}$ content into the fibre is minimised $^{18}$. 


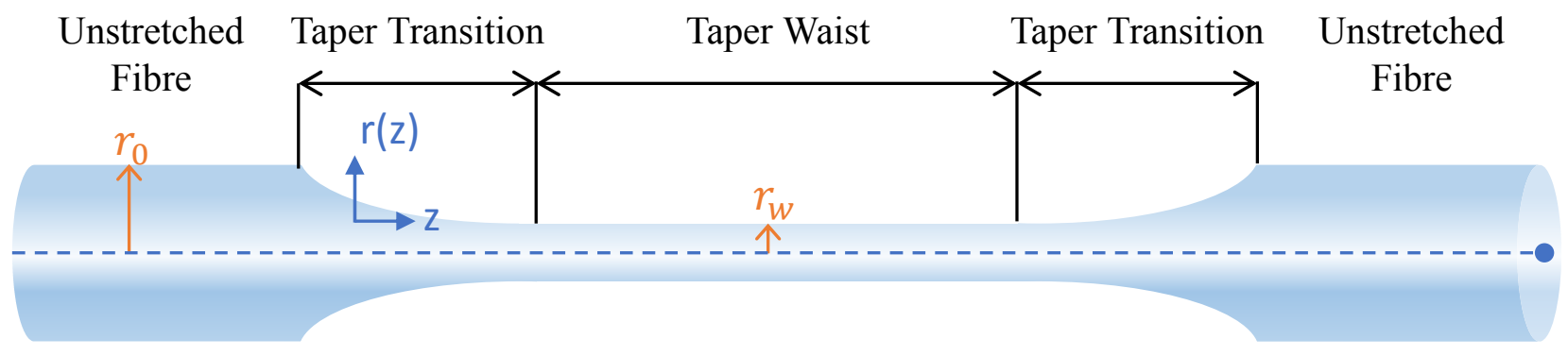

Figure 2. Properties of a tapered optical fibre whose original radius before tapering, $r_{0}$ is shown at the unstretched region, and the thinnest radius, $r_{w}$ at the taper waist.

A single-mode OFS G.652.D fibre with initial diameter $125 \mu \mathrm{m}$ is mechanically stripped, threaded through the microfurnace and spliced with either end fixed to moving stages (Thorlabs DDS220/M). The splice is placed such that it remains outside of the hot zone over the entire tapering process. Including a splice in the heated region gives rise to bottle microresonators with defects. The laser is given time to reach output power stability and is kept at a fixed power throughout the tapering procedure. The stages remain stationary for $18 \mathrm{~s}$ after the laser shutter is opened while the microfurnace is heated to the annealing temperature, after which stages begin the tapering process.

During each cycle of our tapering procedure the fibre is stretched $2 \mathrm{~mm}$ by unidirectional stage movement, firstly in the positive $\mathrm{z}$ direction, and then in the negative $\mathrm{z}$ direction. Each half-cycle, the leading stage moves $4 \mathrm{~mm}$ and the trailing stage moves $3 \mathrm{~mm}$ in the positive $\mathrm{z}$ direction using different fixed velocities to complete the movement in the same time interval. The stages then reverse direction and repeat the movement in the negative $\mathrm{z}$ direction, with the leading stage becoming the trailing stage, to complete a full cycle.

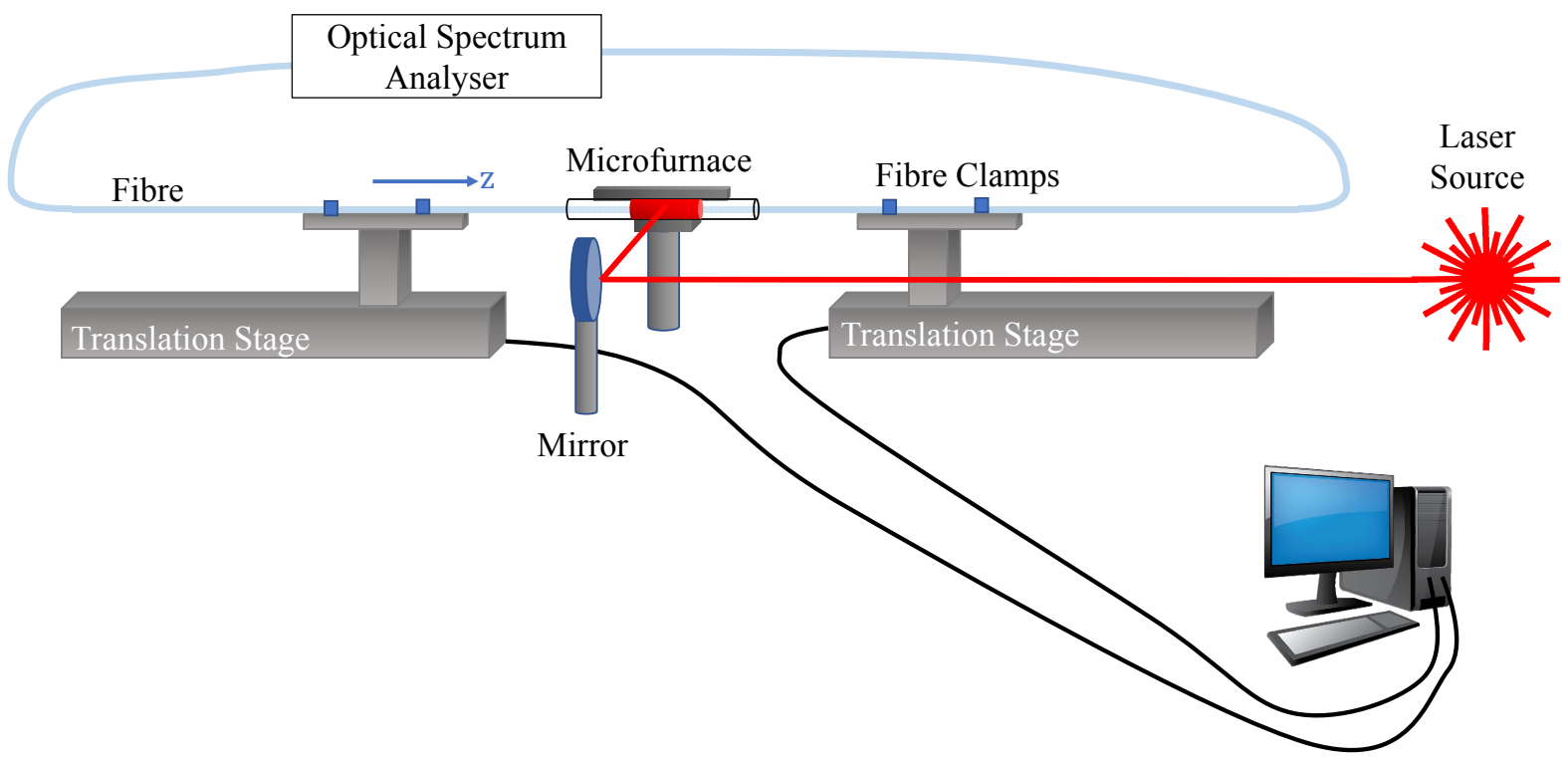

Figure 3. Tapering setup with a $\mathrm{CO}_{2}$ laser source heating a sapphire microfurnace. Computer controlled translation stages pull a standard fibre to the predetermined diameter. Transmission of light through the fibre during tapering is monitored using an OSA with an embedded laser source. 


\subsection{Characterisation}

After tapering, we estimate our waist radius assuming constant fibre density and a uniform taper waist,

$$
r_{w}=r_{0}\left(\frac{L_{0}}{L_{0}+\delta z}\right)^{n}
$$

where $L_{0}$ is the length of fibre heated by the laser beam ( $3 \mathrm{~mm}$, as set by the distance travelled by the trailing stage), $\delta z$ is the extension per half cycle $(1 \mathrm{~mm})$ and $n$ is the number of cycles. Our taper fabrication used 5 cycles giving $r_{w}=14.8$ $\mu \mathrm{m}$.

The subsequent tapered region is characterised using WGM spectroscopy using a probe taper of $\sim 1.7 \mu \mathrm{m}$ diameter waist and optical spectrum analyser (LUNA OVA 5000) with resolution $1.3 \mathrm{pm}$, see Fig. 4. The probe taper is placed at equally-spaced positions, perpendicular to the taper under test (TUT), and measures the power transmission over a $\sim 40$ $\mathrm{nm}$ spectral bandwidth with a central wavelength of $1550 \mathrm{~nm}$.

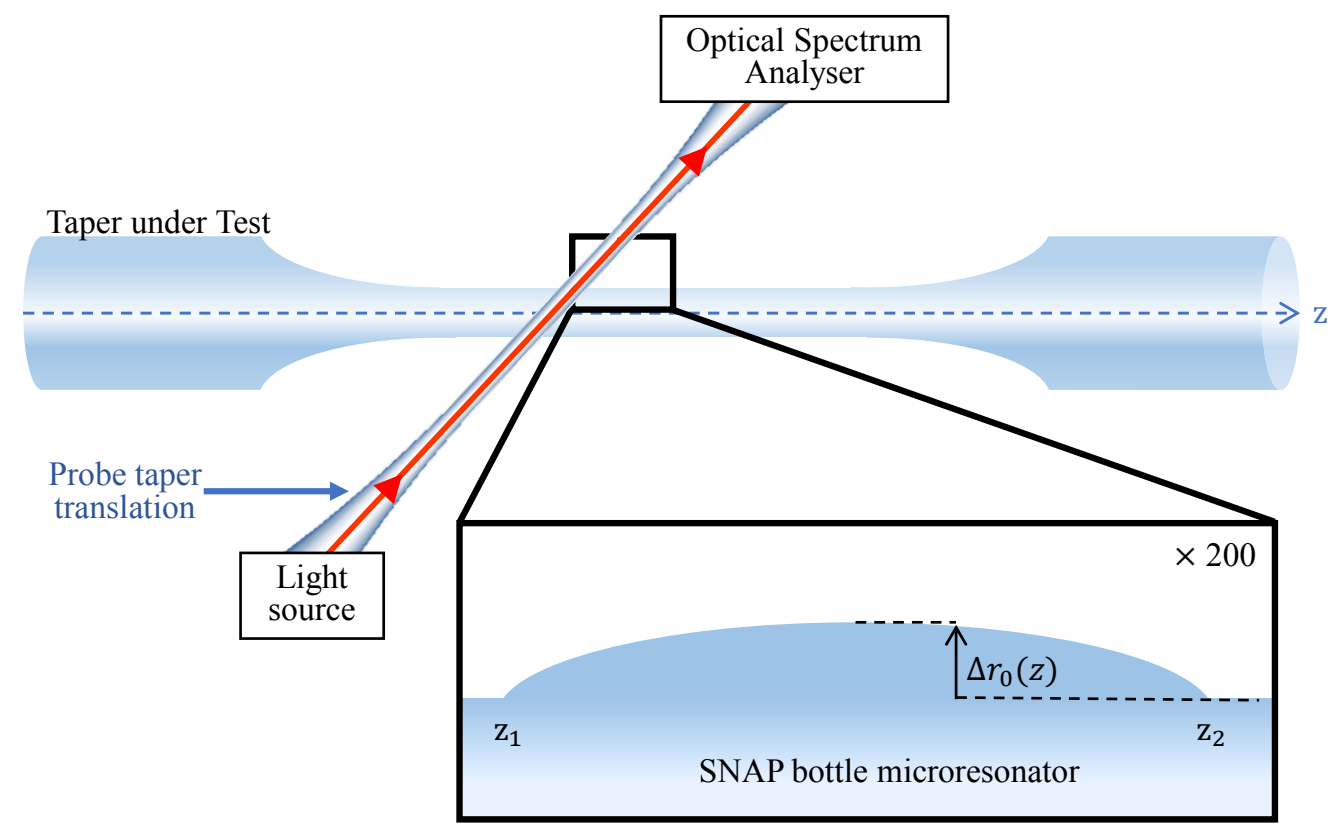

Figure 4. A fabricated taper is tested using WGM spectroscopy with a micron-diameter probe taper placed at different positions along the taper under test. Inset is $\times 200$ zoomed figure of a SNAP bottle microresonator in the taper waist between $\mathrm{z}_{1}$ and $\mathrm{z}_{2}$, with ERV height, $\Delta r_{0}(z)$.

The transmission output has a broad spectrum with dips at wavelengths corresponding to microresonator modes. The collated spectral measurements from different probe taper positions make a spectrogram from which the spatial profile of the ERV along the tapered region of the fibre is found. ERV is proportional to the resonant wavelength variation $\Delta \lambda$ by the rescaling relation ${ }^{16}$,

$$
\Delta r_{0}=r_{w} \cdot \frac{\Delta \lambda}{\lambda_{r}}
$$

where TUT radius waist $r_{w}=14.8 \mu \mathrm{m}$ and $\lambda_{r}$ is the resonant wavelength of the TUT.

In addition to the resonator spatial profile, the spectrogram measures multiple orders of WGMs, allowing determination of characteristic axial and azimuthal FSRs. As mentioned, axial eigenfrequencies may be found analytically by solving the 1D Schrödinger equation. 


\section{ANALYSIS OF EXPERIMENTAL DATA}

\subsection{Taper Waist Spectrograms}

We present two exemplar tapers, A and B, with parabolic resonators in their waist regions. Here we define the taper waist limit at the troughs of our spectrogram, beyond which the steep rates of change indicate the transition regions towards unstretched fibre (see Fig. 2). The range of the transmission power values is determined by the coupling between the probe taper and the TUT, set by the diameter of the probe at the point of contact and selected by the transverse positioning of the probe.

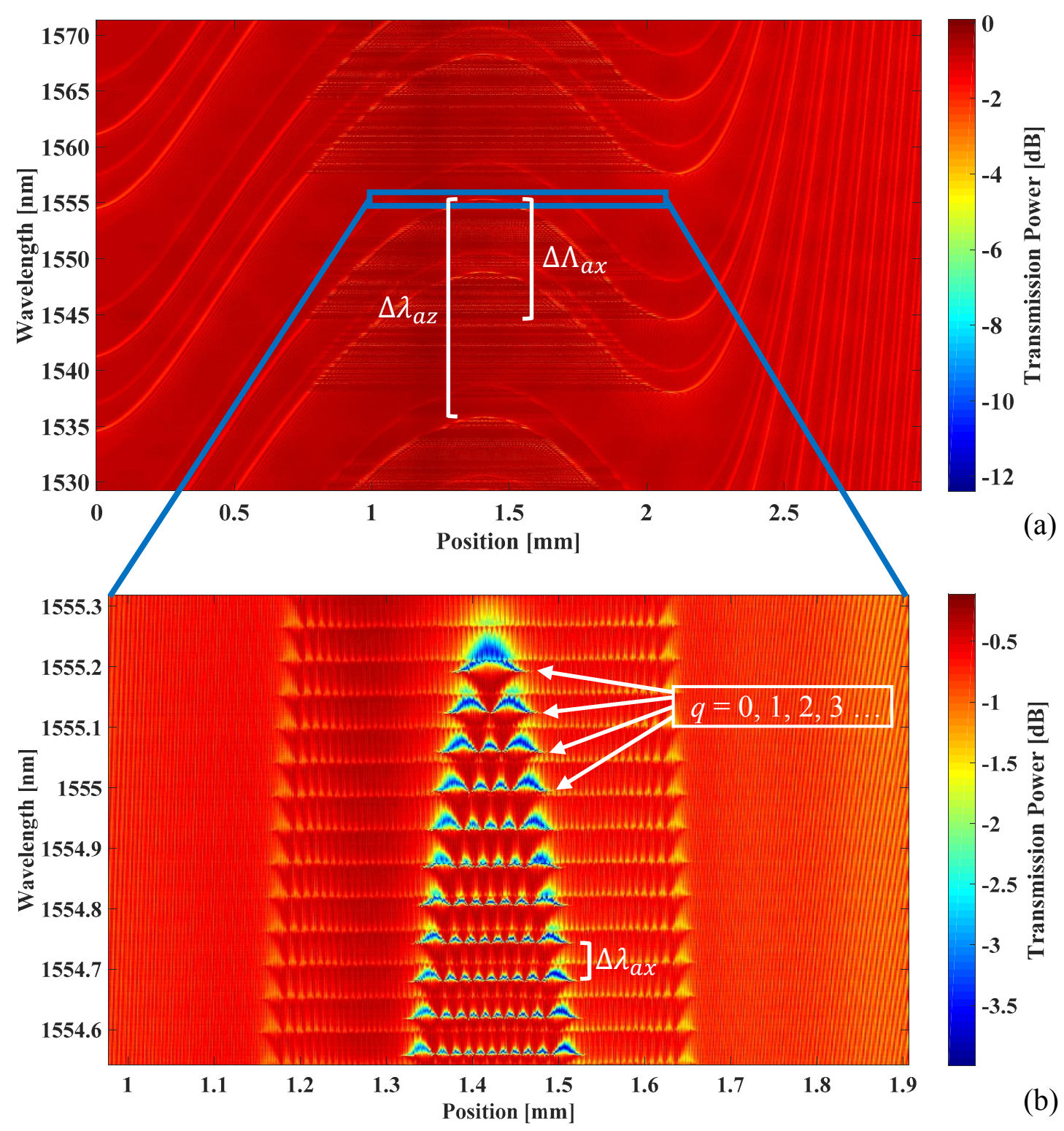

Figure 5. (a) Spectrogram of Taper A where probe translation step size is $2 \mu \mathrm{m}$. Indicated in white is FSR between periodic azimuthal modes, $\Delta \lambda_{a z}$ and bandwidth of axial series, $\Delta \Lambda_{a x}$. Other azimuthal modes with different radial quantum numbers and polarisations result in crowding of modes. (b) Detailed spectrogram of parabolic mode structure shown by blue box in (a), labelled with axial mode numbers in white and adjacent resonant mode spacing (FSR), $\Delta \lambda_{a x}$ indicated in white. 




a)

Figure 6. (a) Spectrogram of Taper B where probe translation step size is $4 \mu \mathrm{m}$. Black lines illustrate the defined regions of taper waist and microresonator extents. Background noise accounts for the banding due to dust particles on the probe taper. (b) Detailed spectrogram of parabolic mode structure shown by blue box in (a) in which we see 10 modes overlap thus $\Delta \Lambda_{a x}>\Delta \lambda_{a z}$.

Axial series over large bandwidths of $10 \mathrm{~nm}$ and $19.3 \mathrm{~nm}$ for Tapers A and B respectively, lead to crowding of azimuthal and radial modes. Taper B in Fig. 6 (b) demonstrates spread of one axial series into the adjacent azimuthal series. The capability to generate axial series with a total bandwidth that exceeds the azimuthal FSR is a necessary step towards utilization of multiple azimuthal orders for broader comb bandwidth. The azimuthal FSR is not matched to be an integer multiple of the axial FSR, which would achieve equidistance across multiple azimuthal orders, but the requisite ERV height necessary for this matching is achieved. 


\subsection{Comparison of Microresonator with Theoretical Ideal Parabola}

Single microresonators isolated from spectrograms in Fig. 5 (a) and Fig. 6 (a) are compared with an ideal parabola, $B\left[1-\frac{1}{a^{2}}\left(z-z_{0}\right)^{2}\right] \cdot \Phi\left(a-\left|z-z_{0}\right|\right)$, where $B$ is the scaling factor, $z$ is the position along the fibre, $z_{0}$ is the position at the parabola peak, $a$ is the half-length of the parabola base and $\Phi$ is the heaviside step function.
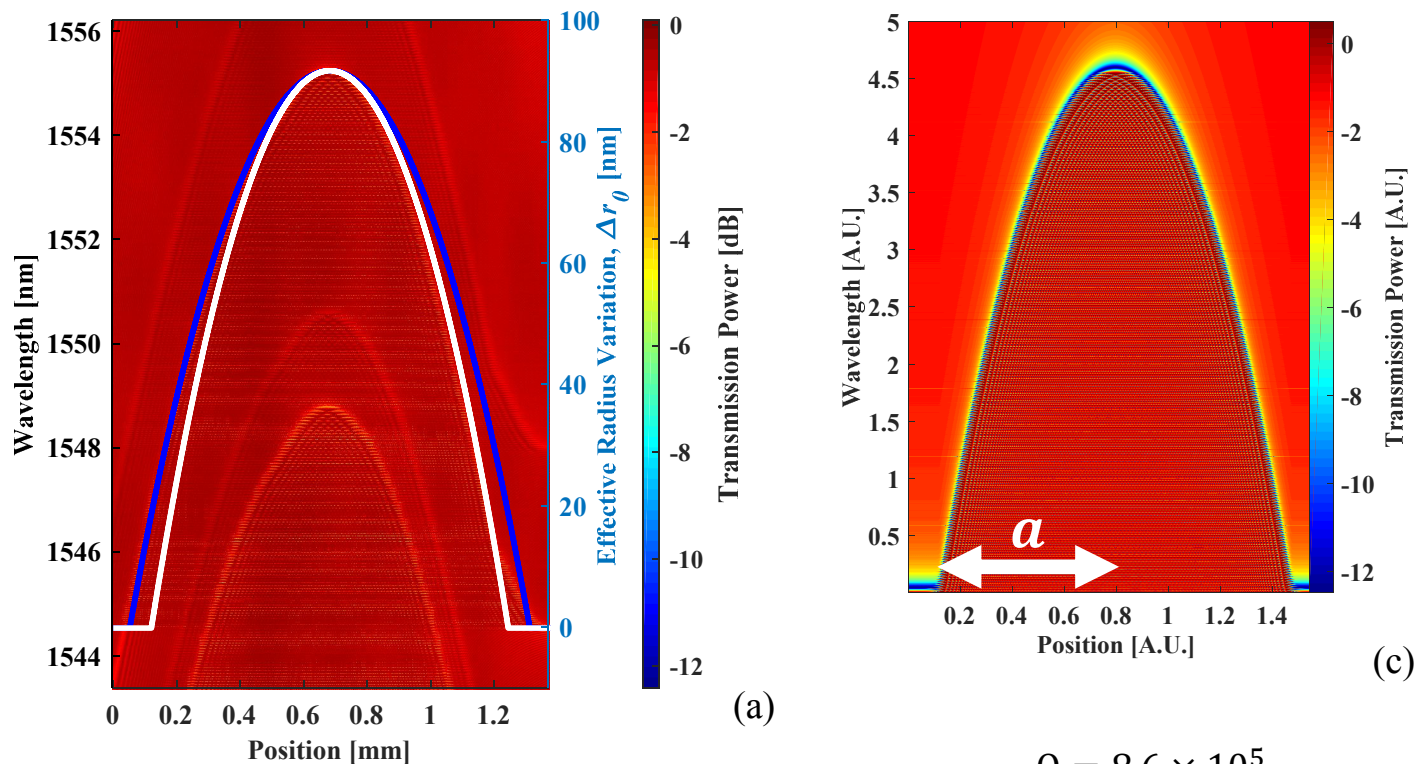

(a)
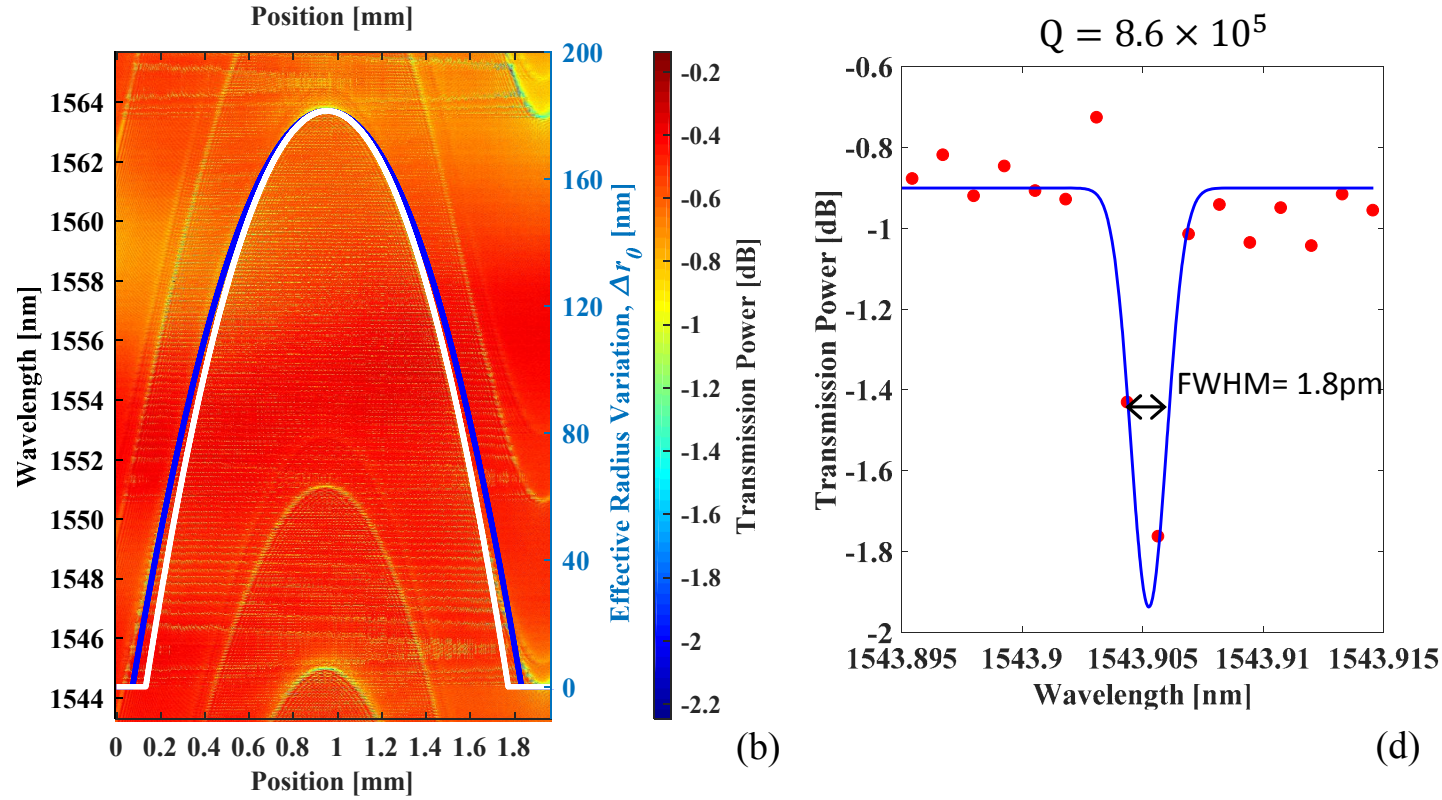

(b)

(d)

Figure 7. (a) Zoomed image of microresonator in Taper A containing hundreds of axial modes. Two ideal parabolic profiles are overlaid: a blue parabola of $a=630 \mu \mathrm{m}$ is fit to left-hand side of the microresonator and an ideal white parabola with $a=562 \mu \mathrm{m}$, is fit to the right-hand side. Each side demonstrates good parabolic fits in the central region of the microresonator, and poor fits at the structure base with the blue parabola also showing poor fit at structure peak. The righthand axis shows the ERV height of the resonant structure. (b) Same as in (a) for Taper B where a blue parabola with $a=880$ $\mu \mathrm{m}$ fit to the left-hand side of the microresonator and a white parabola of $a=862 \mu \mathrm{m}$ is fit to the right-hand side. Each side demonstrates a good parabolic fit in the central region of the microresonator and a poor fit at the structure base. (c) Theoretical model of Taper A using the 1D Schrödinger equation. White arrow illustrates the defined region of $a$, the halflength of the parabola base. (d) Loaded Q-factor calculation using Eq. (4) taken from axial mode $q=90$ of Taper A at outside edge where $\mathrm{z}=1.01 \mathrm{~mm}$. 
The radius of curvature, $R_{0}$, of a parabola is calculated using the relationship ${ }^{10}, y(z)=-A z^{2}$ where $A=\frac{1}{2 R_{0}}$, since $\Delta r_{0}=\frac{a^{2}}{2 R_{0}}$, substituting values for the left parabola fits for both tapers gives $R_{0}=2.10 \mathrm{~m}$ for Taper $\mathrm{A}$ and $R_{0}=2.07 \mathrm{~m}$ for Taper $\mathrm{B}$.

Deviations in the microresonator profiles from ideal parabolas are prominent at the base (at high eigenvalues) for both Taper A and B, as well as at the top of the structure (at low eigenvalues) for Taper A. Tapers A and B show a parabola of larger half-length, fit to the left-hand side of the resonator compared to the right-hand fit. Taper B has significantly closer parabola half-lengths indicating better symmetry under $\mathrm{z}$ parity about the microresonator centre, and in addition has better centring of the microresonator within the taper waist region, shown in Fig. 6 (a). Comparably, the microresonator in Taper A is dramatically off centre within the taper waist, shown in Fig. 5 (a).

\subsection{Axial Mode Spacing}

In order to investigate the equidistance of our eigenfrequencies, below are plots of the axial FSR over a whole series after conversion into frequency domain. We expect ideal parabolas to demonstrate equidistant frequency spacing between modes.

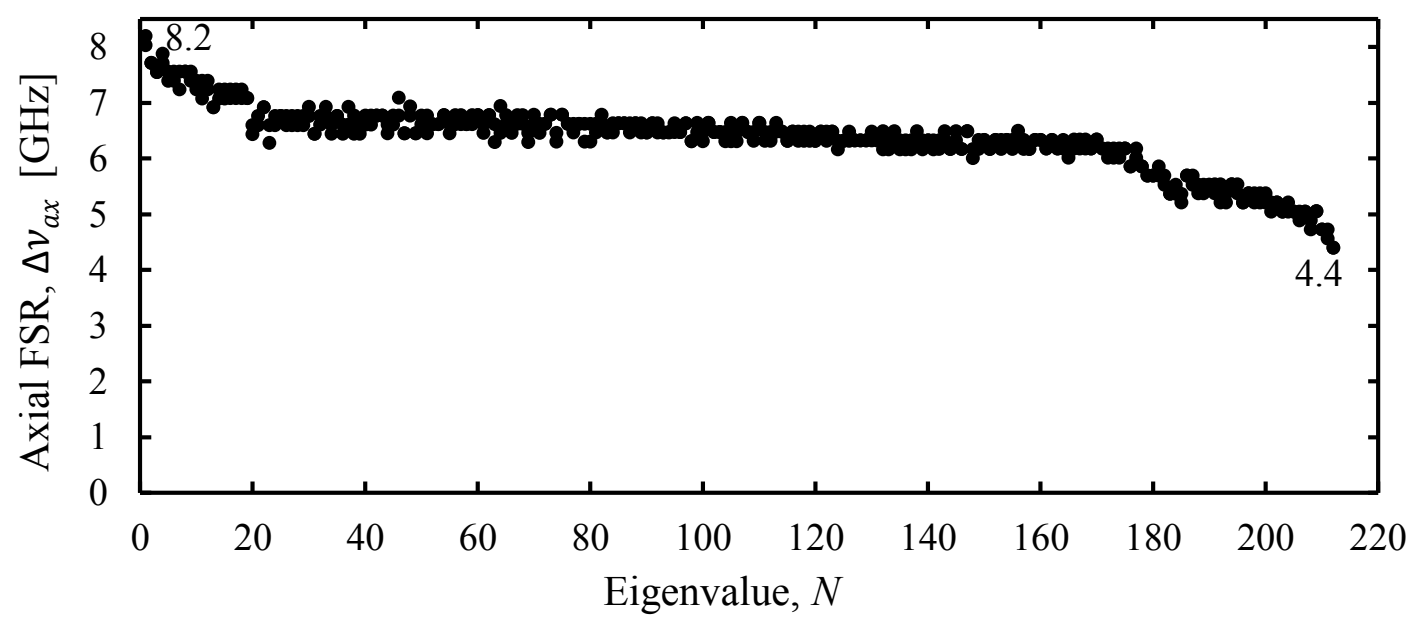

Figure 8. Taper A- mode spacing shows good uniformity for $30<N<170$. Spacing increases for low eigenvalues and decreases for high eigenvalues. Extreme values are labelled giving a spread of $\sim 6 \mathrm{GHz}$ over 213 modes.

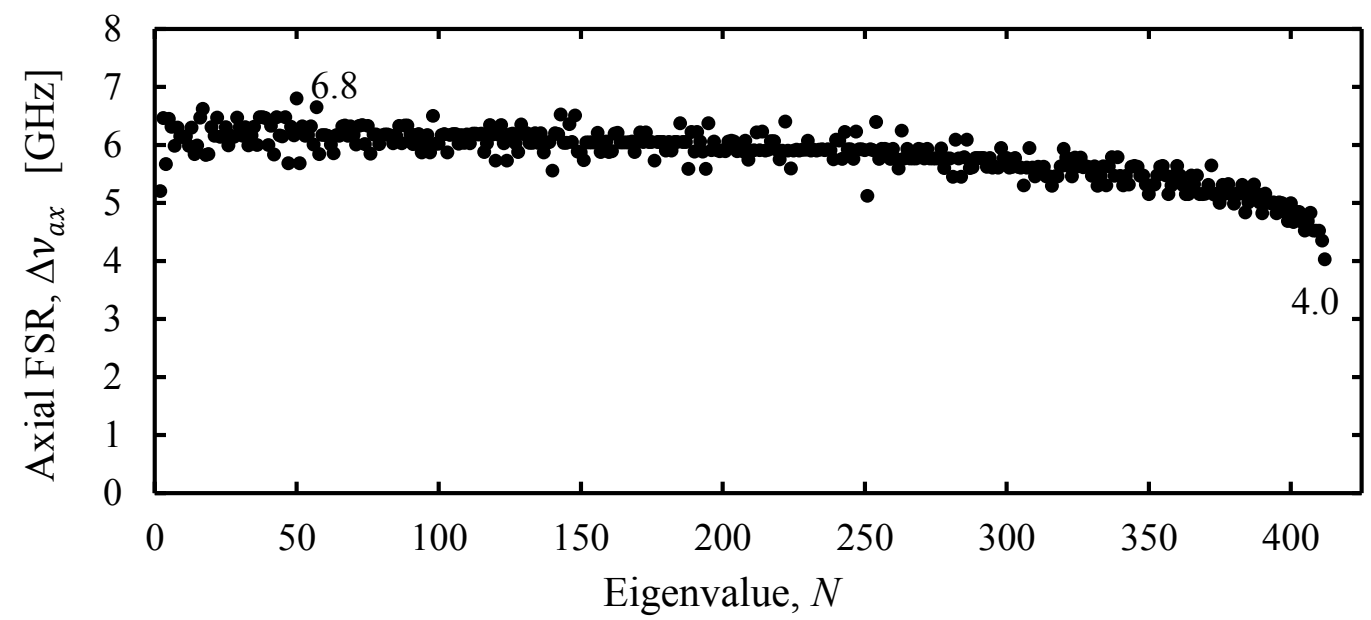

Figure 9. Taper B- mode spacing shows good uniformity for $0<N<280$ after which spacing decreases for high eigenvalues. Extreme values are labelled giving a spread of $\sim 3 \mathrm{GHz}$ over 412 modes. 
Table 1. Taper waist and microresonator characteristics, found using spectrograms. Both tapers underwent 5 cycles during the tapering procedure.

\begin{tabular}{|c|cc|ccccccc|}
\hline & \multicolumn{2}{|c|}{ Taper Waist } & \multicolumn{7}{|c|}{ Micro-Bottle Resonator } \\
\cline { 2 - 10 } & $\begin{array}{c}\text { Extent } \\
{[\mathrm{mm}]}\end{array}$ & $\begin{array}{c}\Delta \lambda_{a z} \\
{[\mathrm{~nm}]}\end{array}$ & $\begin{array}{c}\text { No. of } \\
\begin{array}{c}\text { Axial } \\
\text { Modes }\end{array}\end{array}$ & $\begin{array}{c}\text { Mean } \\
\Delta v_{a x} \\
{[\mathrm{GHz}]}\end{array}$ & $\begin{array}{c}\text { St. Dev. } \\
\Delta v_{a x}\end{array}$ & $\begin{array}{c}\text { Bandwidth } \\
\Delta \Lambda_{a x} \\
{[\mathrm{THz}]}\end{array}$ & $\begin{array}{c}\text { ERV } \\
\Delta r_{0} \\
{[\mathrm{~nm}]}\end{array}$ & $\begin{array}{c}\text { Extent } \\
{[\mathrm{mm}]}\end{array}$ & $\begin{array}{c}\text { Radius of } \\
\text { Curvature, } R_{0} \\
{[\mathrm{~m}]}\end{array}$ \\
\hline Taper A & 2.1 & 19.6 & 213 & 6.4 & $9 \%$ & 1.3 & 96 & 1.4 & 2.10 \\
Taper B & 2.2 & 19.0 & 412 & 5.8 & $7 \%$ & 2.4 & 184 & 1.9 & 2.07 \\
\hline
\end{tabular}

\subsection{Semiclassical Eigenmode Calculation}

The semiclassical asymptotic expression of azimuthal and radial eigenmodes ${ }^{19,20}$ is,

$$
\lambda_{m p}^{ \pm} \approx \frac{2 \pi n_{r} r_{w}}{m}\left(1+\zeta_{p}\left(2 m^{2}\right)^{-\frac{1}{3}}+\frac{n_{r}^{ \pm 1}}{m\left(n_{r}^{2}-1\right)^{\frac{1}{2}}}\right)
$$

where $\zeta_{1,2,3 \ldots}=2.338,4.088,5.521 \ldots$ are the Airy function roots, \pm corresponds to TE/TM mode polarization and $n_{r}=1.46$ refractive index of the bottle resonator material.

Referring to our experimental eigenmode wavelengths the azimuthal and radial FSRs are found for large azimuthal number $m \gg p$, with TE polarization,

$$
\begin{aligned}
\Delta \lambda_{a z_{(97,0)-(98,0)}} & =17 \mathrm{~nm} \\
\Delta \lambda_{\operatorname{rad}_{(97,1)-(97,0)}} & =92 \mathrm{~nm} .
\end{aligned}
$$

Here we see that the radial FSR is 5 times larger than the azimuthal FSR. The corresponding experimental azimuthal measurements of $\sim 19 \mathrm{~nm}$ are in good agreement considering the estimated waist radius used from Eq. (7) assumes a constant fibre density and uniform taper waist during the tapering process.

Broadband and low repetition rate combs require a continuous spectrum such that $\Delta \Lambda_{a x} \geq \Delta \lambda_{a z}$ (note Dvoyrin and Sumetsky refer to $\Delta \Lambda_{a x}$ in frequency, here we refer to wavelength bandwidth). The ERV condition ${ }^{10}$ that the fabricated microresonator should meet is

$$
\Delta r_{0} \geq \frac{\lambda_{0}}{2 \pi n_{r}} \text {. }
$$

For $\lambda_{0}=1550 \mathrm{~nm}, \Delta r_{0} \geq 168 \mathrm{~nm}$, which from Table 1, is satisfied by Taper B with $\Delta r_{0}=184 \mathrm{~nm}$ (calculated using Eq. (8), $\left.\Delta r_{0}=r_{w} \cdot \frac{\Delta \Lambda_{a x}}{\lambda_{r}}\right)$. This agrees with the overlap of modes seen in Fig. 6 (b).

\subsection{Analysis Summary and Discussion}

Our technique of laser brushed microfurnace tapering generates microresonators with parabolic profiles that have large radii of curvature $(\sim 2.1 \mathrm{~m})$ and a repeatable taper waist extent of $\sim 2 \mathrm{~mm}$. The ERV height of both microresonators in Tapers A and B is much larger $(\times 10)$ than typical SNAP resonator devices and their support of hundreds of nearly equidistant axial modes sets a new precedent. We find more modes and a broader longitudinal extent when the microresonator is centred in the waist region. For similar waist extents, the well centred microresonator at Taper B has an ERV height of $184 \mathrm{~nm}$, almost twice as large as Taper A's $96 \mathrm{~nm}$, which along with a larger longitudinal extent produces twice as many axial modes, 412 and 213 respectively.

We consider that the centring of the microresonator in the taper waist region also influences parabolic symmetry. Semiparabolas are fit to each side of the microresonators as shown in Fig. 7 (a)(b), and the difference in parabolic half-lengths is greater for $\mathrm{A}, \Delta a=68 \mu \mathrm{m}$ than for $\mathrm{B}, \Delta a=20 \mu \mathrm{m}$. In Sec. 5.3, axial eigenmode spacing is investigated towards 
assessment of equidistance. Taper A demonstrates mean axial FSR $\Delta v_{a x}=6.4 \mathrm{GHz}$ with a relative standard deviation of $9 \%$ over 213 modes, and Taper B has a mean $\Delta v_{a x}=5.8 \mathrm{GHz}$ with a relative standard deviation of $7 \%$ over 412 modes. In view of Taper B containing twice as many axial modes this clearly demonstrates a superior parabolic profile for Taper B.

Equidistant axial eigenfrequencies are well-suited for dispersion-free delay lines, and post-processing, e.g. addition of a hole to the centre of these parabolic profiles would make a desirable semiparabolic profile. This would halve the microresonator extent to $\sim 0.8 \mathrm{~mm}$ for our exemplar tapers, significantly less than previous semiparabola fabricated on the SNAP platform with $3 \mathrm{~mm}$ extent, whose proportional relation to delay time generates a reasonable few nanoseconds 5 . Therefore, optimization of this technology for delay lines would require augmentation of microresonator extent and reduction of the ERV height.

Full parabolic profiles are directly applicable to frequency comb generation. In particular, Taper B fulfils the condition for the axial series bandwidth to be greater than or equal to the azimuthal FSR. Further effort to match this FSR to be an integer multiple of the axial FSR would broaden the available spectrum substantially by allowing the comb to extend across multiple azimuthal orders. Reduction in fibre radius increases mode spacing and an analytical model could be generated using Eq. (7) for a given bandwidth. This could be used to optimise microresonators for frequency comb generation as the tapering procedure allows predetermination of taper waist radius.

Fabrication of both exemplar tapers follows the same tapering procedure of 5 cycles; nevertheless, large variations in the parabolic profiles are found. ERV height and axial FSR over a whole microresonator series are highly sensitive to how well centred the microresonator is in the taper waist region. Further investigation into the fine tuning of this technique for profile control should include evaluation of pulling parameters including speed, distance and stage symmetry.

\section{CONCLUSIONS}

An alternative method for fabrication of SNAP devices based on optical fibre tapering has been discovered. The fabrication of an optical fibre taper with a $30 \mu \mathrm{m}$ diameter waist from a commercial $125 \mu \mathrm{m}$ diameter, single-mode fibre by laser brushing creates a SNAP bottle microresonator with parabolic radius variation in the centre of the tapered region. Ideal parabolic resonators should demonstrate equal spacing between resonances. Spectral measurement of the parabolic profile shows spacing of $\sim 6 \mathrm{GHz}$ with $10 \%$ deviation over a bandwidth of $2.5 \mathrm{THz}$ containing hundreds of axial modes. Joining these equidistant axial eigenfrequencies using different azimuthal modes indicate a promising avenue to explore for broadband frequency comb generation. Further investigations will explore scaling of the parabolic feature with tapering process parameters, repeatability testing, and the fabrication of more complex shapes.

\section{ACKNOWLEDGMENTS}

The authors acknowledge the support from the Engineering and Physical Sciences Research Council (EPSRC) (EP/P006183/1), Horizon 2020 Framework Programme (H2020-EU.1.3.3, 691011), and US Army Research Laboratory (W911NF-17-2-0048). M. Sumetsky acknowledges the Royal Society Wolfson Research Merit Award (WM130110).

\section{REFERENCES}

[1] Del'Haye, P., Schliesser, A., Arcizet, O., Wilken, T., Holzwarth, R. and Kippenberg, T. J., "Optical frequency comb generation from a monolithic microresonator," Nature 450(7173), 1214-1217 (2007).

[2] Kippenberg, T. J., Holzwarth, R., and Diddams, S. A., "Microresonator-based optical frequency combs," Science 332(6029), 555-559 (2011).

[3] Savchenkov, A. A., Matsko, A. B., Liang, W., Ilchenko, V. S., Seidel, D. and Maleki, L., "Kerr combs with selectable central frequency," Nature Photon. 5(5), 293-296 (2011).

[4] Li, J., Lee, H., Chen, T. and Vahala, K. J., "Low-pump-power, low-phase-noise, and microwave to millimeterwave repetition rate operation in microcombs," Phys. Rev. Lett. 109(23), 1-5 (2012).

[5] Sumetsky, M., "Delay of light in an optical bottle resonator with nanoscale radius variation: Dispersionless, broadband, and low loss," Phys. Rev. Lett., 111(16), 1-5 (2013). 
[6] Sumetsky, M., "Microscopic optical buffering in a harmonic potential," Sci. Rep. 5(1), 1-10 (2015).

[7] Sumetsky, M., "Whispering-gallery-bottle microcavities: the three-dimensional etalon," Opt. Lett. 29(1), 8-10 (2004).

[8] Pöllinger, M., O'Shea, D., Warken, F. and Rauschenbeutel A., "Ultrahigh-Q tunable whispering-gallery-mode microresonator," Phys. Rev. Lett. 103(5), 1-4 (2009).

[9] Toropov, N. A. and Sumetsky, M., "Permanent matching of coupled optical bottle resonators with better than $0.16 \mathrm{GHz}$ precision," Opt. Lett. 41(10), 2278-2281 (2016).

[10] Dvoyrin, V. and Sumetsky, M., "Bottle microresonator broadband and low-repetition-rate frequency comb generator," Opt. Lett. 41(23), 5547-5550 (2016).

[11]Birks, T. A. and Li, Y. W., "The shape of fiber tapers,” J. Lightwave Technol. 10(4), 432-438 (1992).

[12]Birks, T. A., Knight, J. C. and Dimmick, T. E., "High-resolution measurement of the fiber diameter variations using whispering gallery modes and no optical alignment," IEEE Photon. Technol. Lett. 12(2), 182-183 (2000).

[13] Combrié, S., Lehoucq, G., Moille, G., Martin, A. and De Rossi, A., "Comb of high-Q resonances in a compact photonic cavity," Laser Photon. Rev. 11(6), 1-8 (2017).

[14] Strelow, Ch., Rehberg, H., Schultz, C. M., Welsch, H., Heyn, Ch., Heitmann, D., and Kipp, T., "Optical microcavities formed by semiconductor microtubes using a bottlelike geometry," Phys. Rev. Lett. 101(12), 1-4 (2008).

[15] Sumetsky M., "Nanophotonics of optical fibers,” Nanophotonics 2(5-6), 393-406 (2013).

[16] Sumetsky, M., "Theory of SNAP devices: basic equations and comparison with the experiment," Opt. Express, 20(20), 22537-22554 (2012).

[17] Sumetsky, M., Dulashko, Y. and Hale, A., "Fabrication and study of bent and coiled free silica nanowires: Selfcoupling microloop optical interferemoter," Opt. Express 12(15), 3521-3531 (2004).

[18] Brambilla, G., "Optical fibre nanowires and microwires: a review," J. Opt. 12(4), 1-19 (2014).

[19] Demchenko Y. A. and Gorodetsky M. L., "Analytical estimates of eigenfrequencies, dispersion, and field distribution in whispering gallery resonators," J. Opt. Soc. Am. B 30(11), 3056-3063 (2013).

[20]Hamidfar, T., Tokmakov, K. V., Mangan, B. J., Windeler, R. S., Dmitriev, A. V., Vitullo, D. L. P., Bianucci, P. and Sumetsky, M., "Localization of light in an optical microcapillary introduced by a droplet," Optica 5(4), 382-388 (2018). 